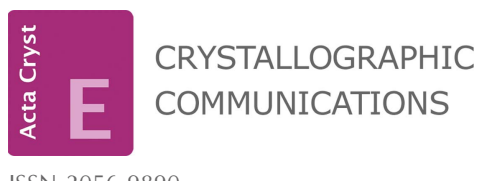

ISSN 2056-9890

\section{Hydrogen bonding, $\pi-\pi$ stacking and van der Waals forces-dominated layered regions in the crystal structure of 4-aminopyridinium hydrogen (9-phosphonononyl)phosphonate}

\author{
Martin van Megen, Guido J. Reiss* and Walter Frank
}

Received 14 August 2016

Accepted 8 September 2016

Edited by W. T. A. Harrison, University of Aberdeen, Scotland

Keywords: crystal structure; 4-aminopyridinium; bis(phosphonate); hydrogen bonding.

CCDC reference: 1503436

Supporting information: this article has supporting information at journals.iucr.org/e

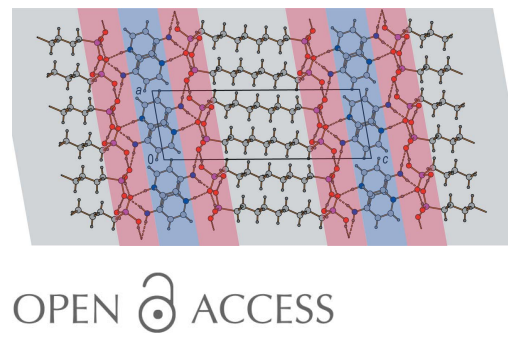

Institut für Anorganische Chemie und Strukturchemie, Lehrstuhl II: Material- und Strukturforschung, Heinrich-HeineUniversität Düsseldorf, Universitätsstrasse 1, D-40225 Düsseldorf, Germany. *Correspondence e-mail: reissg@hhu.de

The asymmetric unit of the title molecular salt, $\left[\mathrm{C}_{5} \mathrm{H}_{7} \mathrm{~N}_{2}{ }^{+}\right]\left[(\mathrm{HO})_{2} \mathrm{OP}\left(\mathrm{CH}_{2}\right)_{9} \mathrm{PO}_{2}{ }^{-}\right.$ $\left.(\mathrm{OH})^{-}\right]$, consists of one 4-aminopyridinium cation and one hydrogen (9-phosphonononyl)phosphonate anion, both in general positions. As expected, the 4-aminopyridinium moieties are protonated exclusively at their endocyclic nitrogen atom due to a mesomeric stabilization by the imine form which would not be given in the corresponding double-protonated dicationic species. In the crystal, the phosphonyl $\left(-\mathrm{PO}_{3} \mathrm{H}_{2}\right)$ and hydrogen phosphonate $\left(-\mathrm{PO}_{3} \mathrm{H}\right)$ groups of the anions form two-dimensional $\mathrm{O}-\mathrm{H} \cdots \mathrm{O}$ hydrogen-bonded networks in the $a b$ plane built from 24-membered hydrogen-bonded ring motifs with the graph-set descriptor $R_{6}^{6}(24)$. These networks are pairwise linked by the anions' alkylene chains. The 4-aminopyridinium cations are stacked in parallel displaced face-to-face arrangements and connect neighboring anionic substructures via medium-strong charge-supported $\mathrm{N}-\mathrm{H} \cdots \mathrm{O}$ hydrogen bonds along the $c$ axis. The resulting three-dimensional hydrogen-bonded network shows clearly separated hydrophilic and hydrophobic structural domains.

\section{Chemical context}

Salts of organophosphonic acids with organic cations, e.g. with protonated primary (Mahmoudkhani \& Langer, 2002b), secondary (Wheatley et al., 2001) and tertiary amines (Kan \& $\mathrm{Ma}, 2011)$ are of growing interest in supramolecular chemistry and crystal engineering. Besides their interesting topologies and structural diversity, they seem to be feasible model compounds for metal phosphonates as they exhibit similar structural characteristics but are less difficult to crystallize. Mostly, these organic solids establish extended hydrogenbonded networks which are characterized by a rich diversity of strong charge-supported hydrogen bonds (Aakeröy \& Seddon, 1993) and can either be one-, two- or three-dimensional. This contribution forms part of our research on the principles of the arrangement of alkane- $\alpha, \omega$-diphosphonic acids (van Megen et al., 2015) and their organic aminium salts (van Megen et al., 2016). Moreover, aminopyridines and the related protonated cations are of crucial interest in the field of biochemistry (Muñoz-Caro \& Niño, 2002; Bolliger et al., 2011) and are also used as counter-cations to stabilize complex salts (Reiss \& Leske, 2014a,b), in crystal engineering (Sertucha et al., 1998; Surbella III et al., 2016) as well as in polymer chemistry (Deng et al., 2015). 
<smiles>Nc1cc[nH+]cc1I</smiles>

Scheme 1

\section{Structural commentary}

The asymmetric unit of the title compound, $\left[\mathrm{C}_{5} \mathrm{H}_{7} \mathrm{~N}_{2}^{+}\right]\left[(\mathrm{HO})_{2} \mathrm{OP}\left(\mathrm{CH}_{2}\right)_{9} \mathrm{PO}_{2}(\mathrm{OH})^{-}\right]$, consists of one 4 -aminopyridinium cation and one hydrogen (9-phosphonononyl)phosphonate anion, both in general positions (Fig. 1). Generally, the first protonation of the 4-aminopyridine can take place at the exo- as well as at the endocyclic nitrogen atom. In the literature, all monoprotonated 4-aminopyridines characterized to date are protonated at the endocyclic nitrogen atom. Geometric parameters derived from the singlecrystal diffraction experiment for the title compound show a short exocyclic $\mathrm{N}-\mathrm{C}$ bond length [1.324 (2) $\mathrm{A}]$ and slightly longer $\mathrm{C}-\mathrm{C}$ and $\mathrm{C}-\mathrm{N}$ bond lengths of the six-membered ring [1.350 (3)-1.425 (2) ̊]. The bonding properties of this cation are best described by a pair of mesomeric structures: the enamine and the imine form (Scheme 2), which have been discussed in detail before (Koleva et al., 2008).<smiles>Nc1cc[nH+]cc1</smiles>

4-aminopyridin-1-ium

Scheme 2
Table 1

Hydrogen-bond geometry $\left(\AA{ }^{\circ}\right)$.

\begin{tabular}{lllll}
\hline$D-\mathrm{H} \cdots A$ & $D-\mathrm{H}$ & $\mathrm{H} \cdots A$ & $D \cdots A$ & $D-\mathrm{H} \cdots A$ \\
\hline $\mathrm{O} 3-\mathrm{H} 3 \cdots \mathrm{O} 6^{\mathrm{i}}$ & $0.78(3)$ & $1.85(3)$ & $2.6171(18)$ & $166(3)$ \\
$\mathrm{O} 5-\mathrm{H} 5 \cdots \mathrm{O} 1^{\text {ii }}$ & $0.88(3)$ & $1.64(3)$ & $2.5059(18)$ & $168(3)$ \\
$\mathrm{O} 4-\mathrm{H} 4 \cdots \mathrm{O} 2^{\text {iii }}$ & $0.91(3)$ & $1.59(3)$ & $2.4977(17)$ & $178(3)$ \\
$\mathrm{N} 1-\mathrm{H} 1 \cdots \mathrm{O} 6$ & $0.96(2)$ & $1.74(3)$ & $2.696(2)$ & $173(2)$ \\
$\mathrm{N} 2-\mathrm{H} 22 \cdots \mathrm{O} 2^{\text {iv }}$ & $0.90(3)$ & $1.92(3)$ & $2.806(2)$ & $170(2)$ \\
$\mathrm{N} 2-\mathrm{H} 21 \cdots 1^{v}$ & $0.88(3)$ & $2.14(3)$ & $2.965(2)$ & $156(3)$ \\
\hline
\end{tabular}

Symmetry codes: (i) $\quad-x+1,-y+2,-z+1 ; \quad$ (ii) $\quad-x,-y+1,-z+1$; (iii) $-x+1,-y+1,-z+1$; (iv) $x, y-1, z+1$; (v) $x+1, y-1, z+1$.

For the designation of the title compound, the systematic name of the amino form is used throughout this article. The bond lengths and angles of the anion are unexceptional and lie within the expected ranges. The alkylene chain of the anion shows nearly antiperiplanar conformations. In detail, the $\mathrm{P}-$ $\mathrm{OH}$ distances of the phosphonate moieties have values between 1.5535 (13) and $1.5786(14) \AA$, longer than the $\mathrm{P}=\mathrm{O}$ distances [1.5045 (13)-1.5149 (12) А].

\section{Supramolecular features}

Within the crystal of the title compound, the phosphonyl and hydrogen phosphonate groups of the anions form twodimensional $\mathrm{O}-\mathrm{H} \cdots \mathrm{O}$ hydrogen-bonded networks which propagate in the $a b$ plane. These networks contain 24membered rings classified as a third level graph set $R_{6}^{6}(24)$ (Etter et al., 1990; Fig. 2; Table 1). 24-Membered hydrogenbonded rings have been well known for decades (e.g. Mootz \& Poll, 1984). In particular, the $R_{6}^{6}(24)$ motif is very common (e.g. Gomathi \& Muthiah, 2011; Maspoch et al., 2007). Along the $c$ axis direction, these networks are pairwise linked by the anions' alkylene chains to form a three-dimensional anionic substructure. The 4-aminopyridinium cations show $\pi-\pi$ stacking interactions. The rings are oriented in parallel displaced face-to-face arrangements (Grimme, 2008; Fig. 3). The geometry of these $\pi-\pi$ interactions is reflected by distances of 3.25 and $3.32 \AA$ between neighbouring pyridinium

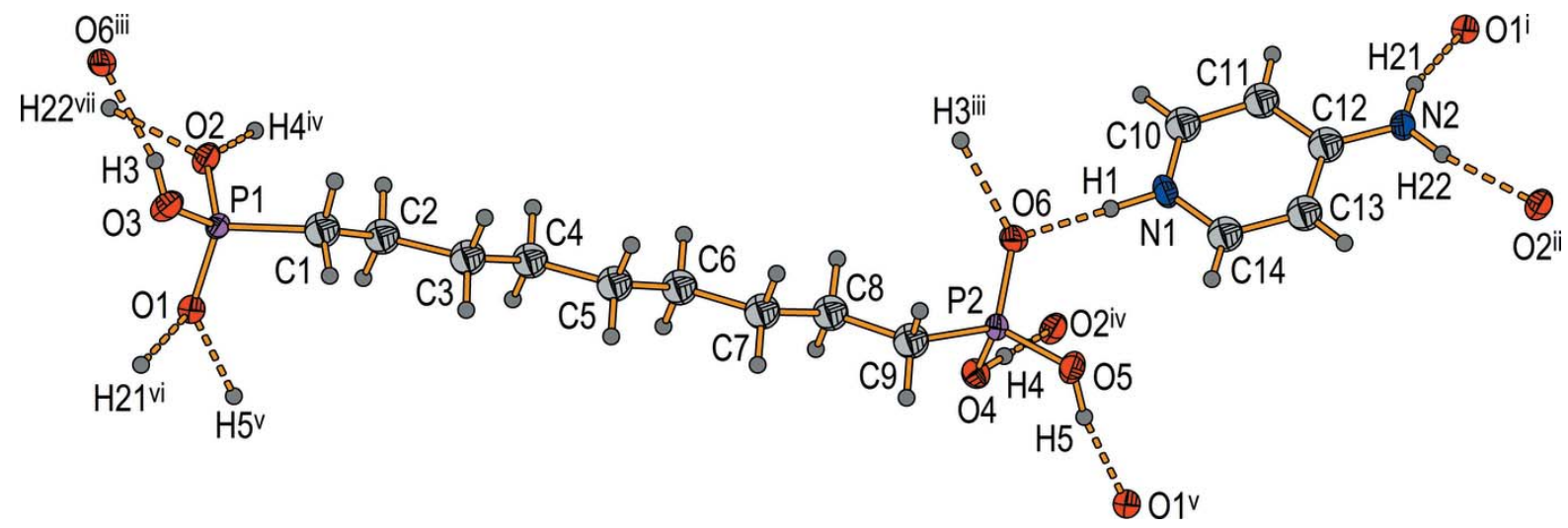

Figure 1

The asymmetric unit of the title compound plus symmetry-related hydrogen-bonded atoms [displacement ellipsoids are drawn at the $50 \%$ probability level; hydrogen atoms are drawn as spheres with arbitrary radii; symmetry codes: (i) $1+x,-1+y, 1+z$; (ii) $x,-1+y, 1+z ;$ (iii) $1-x, 2-y, 1-z$; (iv) $1-x, 1-y, 1-z ;(\mathrm{v})-x, 1-y, 1-z ;(\mathrm{vi})-1+x, 1+y,-1+z,(\mathrm{vii}) x, 1+y,-1+z]$. 


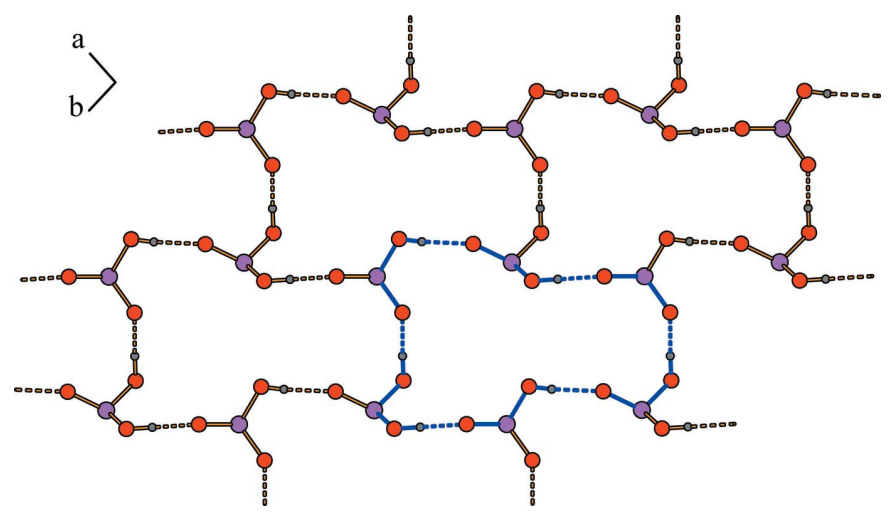

Figure 2

Two-dimensional hydrogen-bonded networks composed of phosphonyl and hydrogen phosphonate groups. The graph set $R_{6}^{6}(24)$ is indicated by blue bonds.

rings and centroid offsets of 2.37 and $2.42 \AA$. These findings are comparable to those found for other compounds containing pyridyl moieties (Janiak, 2000). Anions and cations are connected by medium-strong, charge-supported $\mathrm{N}-$ H...O hydrogen bonds (Steiner, 2002; Table 2) along the $c$ axis. For these connections, each nitrogen-bound hydrogen atom forms one unbifurcated hydrogen bond (Fig. 1). The resulting three-dimensional hydrogen-bonded network clearly shows separated hydrophilic and hydrophobic regions (Fig. 3).

\section{Related structures}

For related phosphonate and bis(phosphonate) salts, see: Ferguson et al. (1998); Fu et al. (2004); Fuller \& Heimer (1995); Glidewell et al. (2000); Kan \& Ma (2011); Mahmoud- khani \& Langer (2002a,b,c); van Megen et al. (2016); Plabst et al. (2009); Wheatley et al. (2001). For related 4-aminopyridinium salts, see: Sertucha et al. (1998); Reiss \& Leske (2014a,b); Surbella III et al. (2016).

\section{Synthesis and crystallization}

Equimolar quantities $(0.5 \mathrm{mmol})$ of 4-aminopyridine (47.1 mg) and nonane-1,9-diphosphonic acid (144.1 mg) were dissolved in methanol, separately. The solutions were mixed and stored in an open petri dish. Within several days, colorless platelet-shaped crystals of the title compound were obtained by slow evaporation of the solvent. 4-Aminopyridine was purchased from commercial sources and nonane-1,9-diphosphonic acid was synthesized according to the literature (Schwarzenbach \& Zurc, 1950; Moedritzer \& Irani, 1961; Griffith et al., 1998). Elemental analysis: $\mathrm{C}_{14} \mathrm{H}_{28} \mathrm{~N}_{2} \mathrm{O}_{6} \mathrm{P}_{2}$ (382.3): calculated C 44.0, H 7.4, N 7.3; found C 43.6, H 7.9, N 7.1. M. p.: $157{ }^{\circ}$ C. The IR and Raman spectra of the title compound are shown in Fig. 4.

\section{Refinement}

Crystal data, data collection and structure refinement details are summarized in Table 2. All hydrogen atoms bound to either nitrogen or oxygen atoms were identified in difference syntheses and refined without any geometric constraints or restraints with individual $U_{\text {iso }}(\mathrm{H})$ values. Carbon-bound hydrogen atoms were included using a riding model (AFIX23 option of the SHELX program for the methylene groups and AFIX43 option for the methine groups).

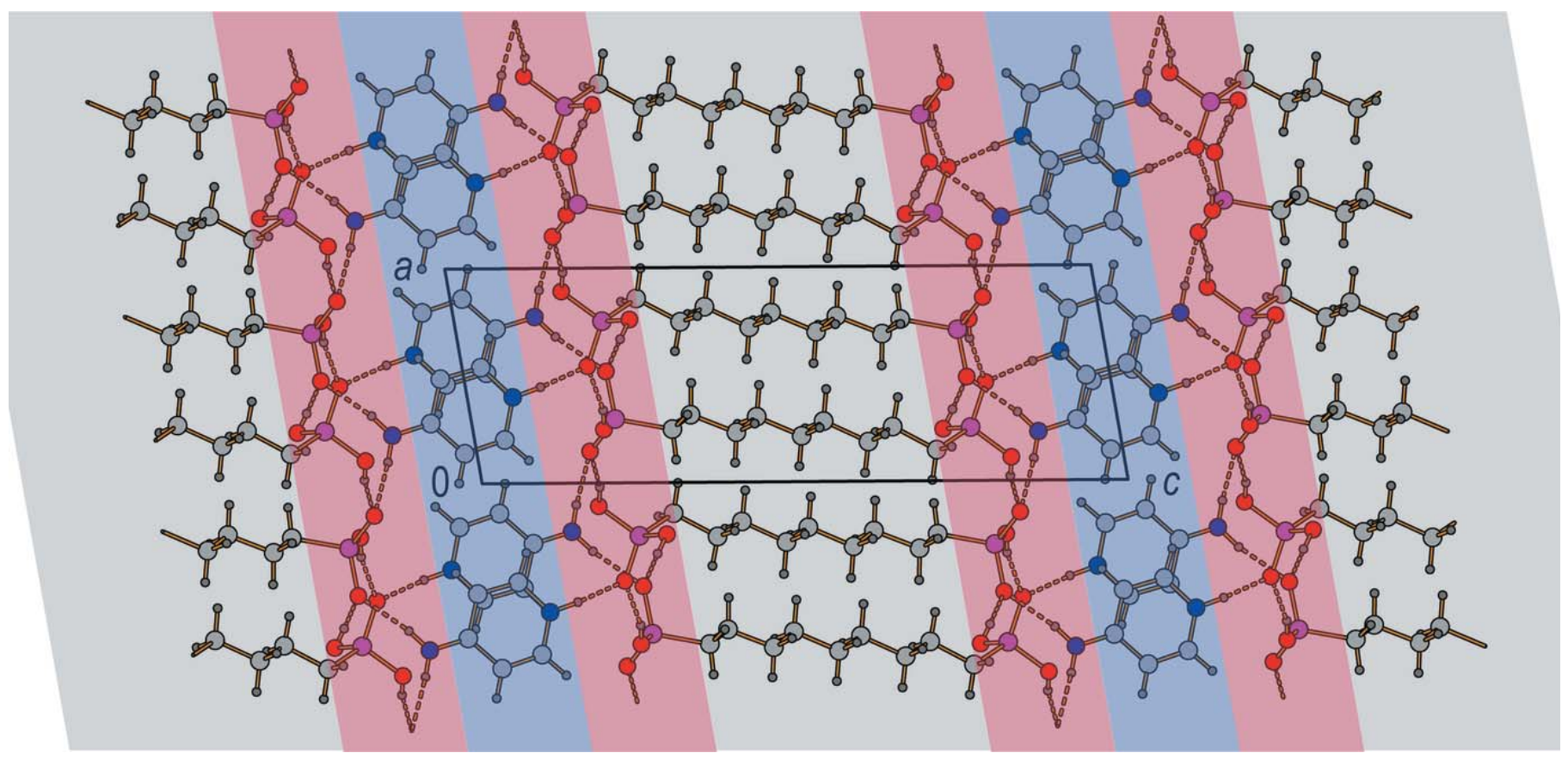

Figure 3

View along [010] of the title structure, showing the hydrogen bonding (red), $\pi-\pi$ stacking (blue), and van der Waals forces (grey) dominated layered regions within the three-dimensional network. 


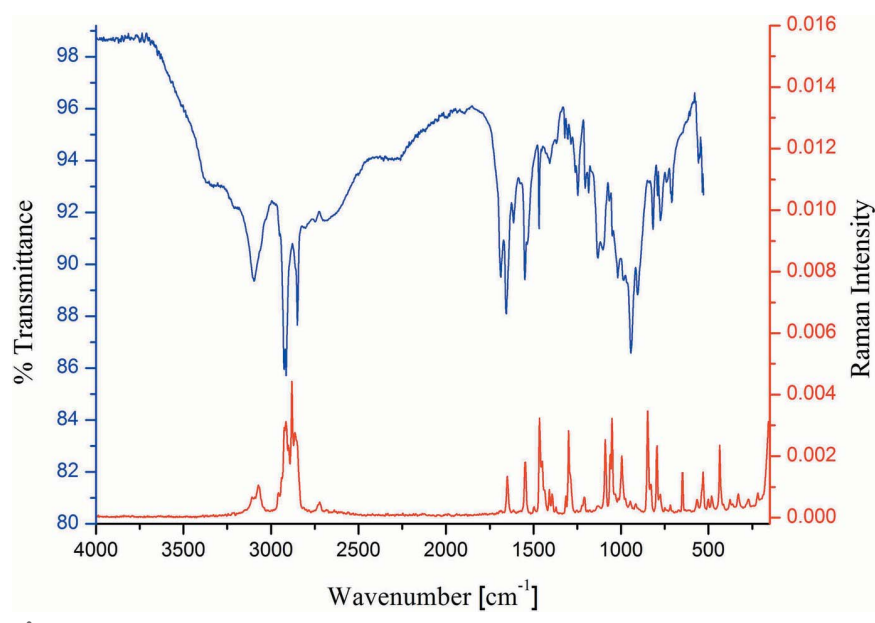

Figure 4

The IR (blue) and Raman (red) spectra of the title compound.

\section{Acknowledgements}

We thank E. Hammes and P. Roloff for technical support.

\section{References}

Aakeröy, C. B. \& Seddon, K. R. (1993). Chem. Soc. Rev. 22, 397-407. Bolliger, J. L., Oberholzer, M. \& Frech, C. M. (2011). Adv. Synth. Catal. 353, 945-954.

Brandenburg, K. (2015). DIAMOND. Crystal Impact GbR, Bonn, Germany.

Deng, Y., Helms, B. A. \& Rolandi, M. (2015). J. Polym. Sci. Part A Polym. Chem. 53, 211-214.

Etter, M. C., MacDonald, J. C. \& Bernstein, J. (1990). Acta Cryst. B46, 256-262.

Ferguson, G., Glidewell, C., Gregson, R. M. \& Meehan, P. R. (1998). Acta Cryst. B54, 129-138.

Fu, R.-B., Wu, X.-T., Hu, S.-M., Du, W.-X. \& Zhang, J.-J. (2004). Chin. J. Struct. Chem. 23, 855-861.

Fuller, J. \& Heimer, N. E. (1995). J. Chem. Crystallogr. 25, 129-136.

Glidewell, C., Ferguson, G. \& Lough, A. J. (2000). Acta Cryst. C56, 855-858.

Gomathi, S. \& Muthiah, P. T. (2011). Acta Cryst. E67, o2762.

Griffith, J. A., McCauley, D. J., Barrans, R. E. \& Herlinger, A. W. (1998). Synth. Commun. 28, 4317-4323.

Grimme, S. (2008). Angew. Chem. Int. Ed. 47, 3430-3434.

Janiak, C. (2000). J. Chem. Soc. Dalton Trans. pp. 3885-3896.

Kan, W.-Q. \& Ma, J.-F. (2011). Z. Kristallogr. New Cryst. Struct. 226, 73-74.

Koleva, B. B., Kolev, T., Seidel, R. W., Tsanev, T., Mayer-Frigge, H., Spiteller, M. \& Sheldrick, W. S. (2008). Spectrochim. Acta part A, 71, 695-702.

Mahmoudkhani, A. H. \& Langer, V. (2002a). Cryst. Growth Des. 2 , 21-25.

Mahmoudkhani, A. H. \& Langer, V. (2002b). J. Mol. Struct. 609, 97108.

Mahmoudkhani, A. H. \& Langer, V. (2002c). Phosphorus, Sulfur Silicon Relat. Elem. 177, 2941-2951.
Table 2

Experimental details.

Crystal data

Chemical formula

$M_{\mathrm{r}}$

Crystal system, space group

Temperature (K)

$a, b, c(\AA)$

$\alpha, \beta, \gamma\left({ }^{\circ}\right)$

$V\left(\AA^{3}\right)$

$Z$

Radiation type

$\mu\left(\mathrm{mm}^{-1}\right)$

Crystal size (mm)

$\mathrm{C}_{5} \mathrm{H}_{7} \mathrm{~N}_{2}{ }^{+} \cdot \mathrm{C}_{9} \mathrm{H}_{21} \mathrm{O}_{6} \mathrm{P}_{2}{ }^{-}$

382.32

Triclinic, $P \overline{1}$

123

6.7275 (4), 6.8963 (4), 20.0643 (10)

97.956 (4), 98.767 (4), 94.309 (5)

$906.73(9)$

2

Mo $K \alpha$

0.27

$0.33 \times 0.07 \times 0.03$

Data collection

Diffractometer

No. of measured, independent and observed $[I>2 \sigma(I)]$ reflections

$R_{\text {int }}$

$(\sin \theta / \lambda)_{\max }\left(\AA^{-1}\right)$

Stoe IPDS

$8855,4131,3674$

0.029

0.650

Refinement

$R\left[F^{2}>2 \sigma\left(F^{2}\right)\right], w R\left(F^{2}\right), S$

No. of reflections

No. of parameters

$\mathrm{H}$-atom treatment

4131

241

$\mathrm{H}$ atoms treated by a mixture of independent and constrained refinement

$\Delta \rho_{\max }, \Delta \rho_{\min }\left(\mathrm{e} \AA^{-3}\right)$ $0.50,-0.36$

Computer programs: X-AREA (Stoe \& Cie, 2002), SHELXT (Sheldrick, 2015a), SHELXL-2014/7 (Sheldrick, 2015b) and DIAMOND (Brandenburg, 2015).

Maspoch, D., Domingo, N., Roques, N., Wurst, K., Tejada, J., Rovira, C., Ruiz-Molina, D. \& Veciana, J. (2007). Chem. Eur. J. 13, 81538163.

Megen, M. van, Frank, W. \& Reiss, G. J. (2015). Z. Kristallogr. 230, 485-494.

Megen, M. van, Frank, W. \& Reiss, G. J. (2016). CrystEngComm, 18, 3574-3584.

Moedritzer, K. \& Irani, R. (1961). J. Inorg. Nucl. Chem. 22, 297-304.

Mootz, D. \& Poll, W. (1984). Z. Naturforsch. Teil B, 39, 290-297.

Muñoz-Caro, C. \& Niño, A. (2002). Biophys. Chem. 96, 1-14.

Plabst, M., Stock, N. \& Bein, T. (2009). Cryst. Growth Des. 9, 50495060.

Reiss, G. J. \& Leske, P. B. (2014a). Z. Kristallogr. New Cryst. Struct. 229, 239-240.

Reiss, G. J. \& Leske, P. B. (2014b). Z. Kristallogr. New Cryst. Struct. 229, 452-454.

Schwarzenbach, G. \& Zurc, J. (1950). Monatsh. Chem. 81, 202-212.

Sertucha, J., Luque, A., Lloret, F. \& Román, P. (1998). Polyhedron, 17, 3875-3880.

Sheldrick, G. M. (2015a). Acta Cryst. A71, 3-8.

Sheldrick, G. M. (2015b). Acta Cryst. C71, 3-8.

Steiner, T. (2002). Angew. Chem. Int. Ed. 41, 48-76.

Stoe \& Cie (2002). $X$-AREA. Stoe \& Cie, Darmstadt, Germany.

Surbella, R. G. III, Andrews, M. B. \& Cahill, C. L. (2016). J. Solid State Chem. 236, 257-271.

Wheatley, P. S., Lough, A. J., Ferguson, G., Burchell, C. J. \& Glidewell, C. (2001). Acta Cryst. B57, 95-102. 


\section{supporting information}

Acta Cryst. (2016). E72, 1456-1459 [https://doi.org/10.1107/S2056989016014298]

Hydrogen bonding, $\pi-\pi$ stacking and van der Waals forces-dominated layered regions in the crystal structure of 4-aminopyridinium hydrogen (9-phosphonononyl)phosphonate

\section{Martin van Megen, Guido J. Reiss and Walter Frank}

Computing details

Data collection: $X$-AREA (Stoe \& Cie, 2002); cell refinement: $X$-AREA (Stoe \& Cie, 2002); data reduction: $X$-AREA (Stoe $\&$ Cie, 2002); program(s) used to solve structure: SHELXT (Sheldrick, 2015a); program(s) used to refine structure:

SHELXL-2014/7 (Sheldrick, 2015b); molecular graphics: DIAMOND (Brandenburg, 2015).

4-Aminopyridinium hydrogen (9-phosphonononyl)phosphonate

\section{Crystal data}

$\mathrm{C}_{5} \mathrm{H}_{7} \mathrm{~N}_{2}^{+} \cdot \mathrm{C}_{9} \mathrm{H}_{21} \mathrm{O}_{6} \mathrm{P}_{2}^{-}$

$M_{r}=382.32$

Triclinic, $P \overline{1}$

$a=6.7275(4) \AA$

$b=6.8963(4) \AA$

$c=20.0643(10) \AA$

$\alpha=97.956(4)^{\circ}$

$\beta=98.767(4)^{\circ}$

$\gamma=94.309(5)^{\circ}$

$V=906.73(9) \AA^{3}$

Data collection

Stoe IPDS

diffractometer

Radiation source: sealed tube

$\omega$ scans

8855 measured reflections

4131 independent reflections

Refinement

Refinement on $F^{2}$

Least-squares matrix: full

$R\left[F^{2}>2 \sigma\left(F^{2}\right)\right]=0.038$

$w R\left(F^{2}\right)=0.079$

$S=1.02$

4131 reflections

241 parameters

0 restraints
$Z=2$

$F(000)=408$

$D_{\mathrm{x}}=1.400 \mathrm{Mg} \mathrm{m}^{-3}$

Mo $K \alpha$ radiation, $\lambda=0.71073 \AA$

Cell parameters from 6853 reflections

$\theta=3.0-35.3^{\circ}$

$\mu=0.27 \mathrm{~mm}^{-1}$

$T=123 \mathrm{~K}$

Platelet, colourless

$0.33 \times 0.07 \times 0.03 \mathrm{~mm}$

3674 reflections with $I>2 \sigma(I)$

$R_{\text {int }}=0.029$

$\theta_{\max }=27.5^{\circ}, \theta_{\min }=3.0^{\circ}$

$h=-8 \rightarrow 8$

$k=-8 \rightarrow 8$

$l=-26 \rightarrow 26$

Secondary atom site location: difference Fourier map

Hydrogen site location: mixed

$\mathrm{H}$ atoms treated by a mixture of independent and constrained refinement

$w=1 /\left[\sigma^{2}\left(F_{\mathrm{o}}^{2}\right)+(0.011 P)^{2}+1.110 P\right]$ where $P=\left(F_{\mathrm{o}}^{2}+2 F_{\mathrm{c}}^{2}\right) / 3$

$(\Delta / \sigma)_{\max }=0.001$

$\Delta \rho_{\max }=0.50$ e $\AA^{-3}$

$\Delta \rho_{\min }=-0.36$ e $\AA^{-3}$ 


\section{Special details}

Geometry. All esds (except the esd in the dihedral angle between two 1.s. planes) are estimated using the full covariance matrix. The cell esds are taken into account individually in the estimation of esds in distances, angles and torsion angles; correlations between esds in cell parameters are only used when they are defined by crystal symmetry. An approximate (isotropic) treatment of cell esds is used for estimating esds involving l.s. planes.

Fractional atomic coordinates and isotropic or equivalent isotropic displacement parameters $\left(\AA^{2}\right)$

\begin{tabular}{|c|c|c|c|c|}
\hline & $x$ & $y$ & $z$ & $U_{\text {iso }} * / U_{\text {eq }}$ \\
\hline P1 & $0.29655(6)$ & $1.15924(6)$ & $0.22320(2)$ & $0.01579(10)$ \\
\hline $\mathrm{O} 1$ & 0.14667 (19) & $1.0094(2)$ & $0.17549(6)$ & $0.0230(3)$ \\
\hline N1 & $0.5995(3)$ & $0.2836(2)$ & $0.92948(8)$ & $0.0257(3)$ \\
\hline H1 & $0.553(4)$ & $0.306(4)$ & $0.8838(13)$ & $0.036(6)^{*}$ \\
\hline $\mathrm{C} 1$ & $0.2470(3)$ & $1.1616(3)$ & $0.30883(8)$ & $0.0179(3)$ \\
\hline $\mathrm{H} 1 \mathrm{~A}$ & 0.3161 & 1.2802 & 0.3371 & $0.021^{*}$ \\
\hline H1B & 0.1032 & 1.1663 & 0.3086 & $0.021^{*}$ \\
\hline P2 & $0.24757(6)$ & $0.29013(6)$ & $0.77082(2)$ & $0.01589(10)$ \\
\hline $\mathrm{O} 2$ & $0.51802(18)$ & $1.13618(18)$ & $0.22159(6)$ & 0.0199 (3) \\
\hline N2 & $0.7740(3)$ & 0.1858 (3) & $1.12583(8)$ & $0.0252(3)$ \\
\hline $\mathrm{H} 21$ & $0.901(4)$ & 0.169 (4) & $1.1403(14)$ & $0.049(8)^{*}$ \\
\hline $\mathrm{H} 22$ & $0.681(4)$ & $0.172(4)$ & $1.1527(12)$ & $0.035(6)^{*}$ \\
\hline $\mathrm{C} 2$ & $0.3143(3)$ & 0.9827 (3) & $0.34082(8)$ & $0.0191(3)$ \\
\hline $\mathrm{H} 2 \mathrm{~A}$ & 0.2686 & 0.8640 & 0.3084 & $0.023^{*}$ \\
\hline $\mathrm{H} 2 \mathrm{~B}$ & 0.4608 & 0.9929 & 0.3504 & $0.023^{*}$ \\
\hline $\mathrm{O} 3$ & $0.2486(2)$ & $1.3660(2)$ & $0.20293(7)$ & $0.0239(3)$ \\
\hline C3 & $0.2306(3)$ & $0.9668(3)$ & $0.40681(8)$ & 0.0191 (3) \\
\hline $\mathrm{H} 3 \mathrm{~A}$ & 0.2699 & 1.0891 & 0.4379 & $0.023^{*}$ \\
\hline H3B & 0.0842 & 0.9503 & 0.3964 & $0.023^{*}$ \\
\hline H3 & $0.343(5)$ & $1.442(5)$ & $0.2068(17)$ & $0.070(11)^{*}$ \\
\hline $\mathrm{O} 4$ & 0.24107 (19) & $0.08343(18)$ & $0.72796(6)$ & $0.0188(2)$ \\
\hline $\mathrm{C} 4$ & $0.3028(3)$ & 0.7975 (3) & $0.44244(8)$ & $0.0195(3)$ \\
\hline $\mathrm{H} 4 \mathrm{~A}$ & 0.4490 & 0.8157 & 0.4542 & $0.023^{*}$ \\
\hline H4B & 0.2664 & 0.6752 & 0.4112 & $0.023^{*}$ \\
\hline $\mathrm{H} 4$ & $0.330(5)$ & $0.006(5)$ & $0.7471(16)$ & $0.066(9)^{*}$ \\
\hline $\mathrm{O} 5$ & $0.1083(2)$ & $0.27587(19)$ & $0.82576(6)$ & $0.0216(3)$ \\
\hline $\mathrm{C} 5$ & $0.2125(3)$ & $0.7822(3)$ & $0.50710(9)$ & $0.0195(3)$ \\
\hline $\mathrm{H} 5 \mathrm{~A}$ & 0.2483 & 0.9055 & 0.5379 & $0.023^{*}$ \\
\hline H5B & 0.0664 & 0.7648 & 0.4950 & $0.023 *$ \\
\hline H5 & $0.017(5)$ & $0.174(5)$ & $0.8191(16)$ & $0.064(9)^{*}$ \\
\hline O6 & 0.45679 (19) & 0.37295 (19) & $0.80557(6)$ & $0.0220(3)$ \\
\hline C6 & $0.2806(3)$ & $0.6151(3)$ & $0.54491(8)$ & 0.0188 (3) \\
\hline H6A & 0.4264 & 0.6325 & 0.5579 & $0.023^{*}$ \\
\hline H6B & 0.2450 & 0.4910 & 0.5146 & $0.023^{*}$ \\
\hline $\mathrm{C} 7$ & $0.1838(3)$ & $0.6074(3)$ & $0.60867(8)$ & $0.0179(3)$ \\
\hline H7A & 0.0383 & 0.5858 & 0.5951 & $0.022 *$ \\
\hline H7B & 0.2146 & 0.7341 & 0.6377 & $0.022^{*}$ \\
\hline C8 & $0.2526(3)$ & $0.4476(3)$ & $0.65035(8)$ & $0.0180(3)$ \\
\hline $\mathrm{H} 8 \mathrm{~A}$ & 0.3972 & 0.4716 & 0.6661 & $0.022 *$ \\
\hline
\end{tabular}




$\begin{array}{lllll}\text { H8B } & 0.2261 & 0.3205 & 0.6214 & 0.022^{*} \\ \text { C9 } & 0.1432(3) & 0.4431(3) & 0.71211(8) & 0.0175(3) \\ \text { H9A } & 0.0022 & 0.3966 & 0.6959 & 0.021^{*} \\ \text { H9B } & 0.1488 & 0.5762 & 0.7360 & 0.021^{*} \\ \text { C10 } & 0.7973(3) & 0.2769(3) & 0.95325(10) & 0.0283(4) \\ \text { H10 } & 0.8919 & 0.2928 & 0.9247 & 0.034^{*} \\ \text { C11 } & 0.8604(3) & 0.2470(3) & 1.01867(9) & 0.0272(4) \\ \text { H11 } & 0.9972 & 0.2420 & 1.0341 & 0.033^{*} \\ \text { C12 } & 0.7189(3) & 0.2236(3) & 1.06310(9) & 0.0199(3) \\ \text { C13 } & 0.5127(3) & 0.2384(3) & 1.03659(9) & 0.0214(4) \\ \text { H13 } & 0.4144 & 0.2288 & 1.0642 & 0.026^{*} \\ \text { C14 } & 0.4601(3) & 0.2665(3) & 0.97075(10) & 0.0242(4) \\ \text { H14 } & 0.3249 & 0.2741 & 0.9537 & 0.029^{*}\end{array}$

Atomic displacement parameters $\left(\AA^{2}\right)$

\begin{tabular}{lllllll}
\hline & $U^{11}$ & $U^{22}$ & $U^{33}$ & $U^{12}$ & $U^{13}$ & $U^{23}$ \\
\hline P1 & $0.01293(19)$ & $0.0198(2)$ & $0.0158(2)$ & $0.00022(16)$ & $0.00233(15)$ & $0.00733(16)$ \\
O1 & $0.0224(6)$ & $0.0286(7)$ & $0.0167(6)$ & $-0.0056(5)$ & $0.0023(5)$ & $0.0042(5)$ \\
N1 & $0.0360(9)$ & $0.0236(8)$ & $0.0159(7)$ & $0.0009(7)$ & $-0.0008(6)$ & $0.0036(6)$ \\
C1 & $0.0179(8)$ & $0.0202(8)$ & $0.0161(8)$ & $-0.0003(6)$ & $0.0038(6)$ & $0.0048(6)$ \\
P2 & $0.0175(2)$ & $0.0167(2)$ & $0.01360(19)$ & $-0.00040(16)$ & $0.00111(15)$ & $0.00535(15)$ \\
O2 & $0.0163(6)$ & $0.0239(6)$ & $0.0228(6)$ & $0.0042(5)$ & $0.0057(5)$ & $0.0111(5)$ \\
N2 & $0.0187(8)$ & $0.0392(10)$ & $0.0194(7)$ & $0.0036(7)$ & $0.0035(6)$ & $0.0097(7)$ \\
C2 & $0.0185(8)$ & $0.0237(9)$ & $0.0162(8)$ & $0.0015(7)$ & $0.0030(6)$ & $0.0072(6)$ \\
O3 & $0.0158(6)$ & $0.0267(7)$ & $0.0328(7)$ & $0.0026(5)$ & $0.0047(5)$ & $0.0162(6)$ \\
C3 & $0.0195(8)$ & $0.0229(9)$ & $0.0163(8)$ & $0.0003(7)$ & $0.0047(6)$ & $0.0066(6)$ \\
O4 & $0.0190(6)$ & $0.0194(6)$ & $0.0178(6)$ & $0.0029(5)$ & $0.0014(5)$ & $0.0036(5)$ \\
C4 & $0.0201(8)$ & $0.0239(9)$ & $0.0161(8)$ & $0.0009(7)$ & $0.0038(6)$ & $0.0079(6)$ \\
O5 & $0.0280(7)$ & $0.0206(6)$ & $0.0170(6)$ & $-0.0021(5)$ & $0.0070(5)$ & $0.0041(5)$ \\
C5 & $0.0209(8)$ & $0.0217(8)$ & $0.0169(8)$ & $0.0002(7)$ & $0.0039(6)$ & $0.0063(6)$ \\
O6 & $0.0216(6)$ & $0.0228(6)$ & $0.0202(6)$ & $-0.0045(5)$ & $-0.0032(5)$ & $0.0091(5)$ \\
C6 & $0.0193(8)$ & $0.0221(8)$ & $0.0161(8)$ & $0.0008(7)$ & $0.0041(6)$ & $0.0056(6)$ \\
C7 & $0.0195(8)$ & $0.0199(8)$ & $0.0153(7)$ & $0.0014(6)$ & $0.0029(6)$ & $0.0060(6)$ \\
C8 & $0.0191(8)$ & $0.0200(8)$ & $0.0157(7)$ & $0.0019(6)$ & $0.0027(6)$ & $0.0058(6)$ \\
C9 & $0.0178(8)$ & $0.0198(8)$ & $0.0157(7)$ & $0.0020(6)$ & $0.0027(6)$ & $0.0054(6)$ \\
C10 & $0.0305(10)$ & $0.0338(11)$ & $0.0206(9)$ & $0.0008(8)$ & $0.0077(7)$ & $0.0020(8)$ \\
C11 & $0.0206(9)$ & $0.0390(11)$ & $0.0215(9)$ & $0.0018(8)$ & $0.0038(7)$ & $0.0032(8)$ \\
C12 & $0.0212(8)$ & $0.0192(8)$ & $0.0184(8)$ & $0.0008(7)$ & $0.0025(6)$ & $0.0014(6)$ \\
C13 & $0.0206(8)$ & $0.0207(8)$ & $0.0232(9)$ & $0.0010(7)$ & $0.0039(7)$ & $0.0047(7)$ \\
C14 & $0.0249(9)$ & $0.0206(9)$ & $0.0254(9)$ & $0.0022(7)$ & $-0.0020(7)$ & $0.0046(7)$ \\
& & & & & & \\
\hline & & & & & &
\end{tabular}

Geometric parameters $\left(\AA,{ }^{\circ}\right)$

\begin{tabular}{llll}
\hline $\mathrm{P} 1-\mathrm{O} 1$ & $1.5088(13)$ & $\mathrm{C} 4-\mathrm{H} 4 \mathrm{~A}$ & 0.9700 \\
$\mathrm{P} 1-\mathrm{O} 2$ & $1.5149(12)$ & $\mathrm{C} 4-\mathrm{H} 4 \mathrm{~B}$ & 0.9700 \\
$\mathrm{P} 1-\mathrm{O} 3$ & $1.5786(14)$ & $\mathrm{O} 5-\mathrm{H} 5$ & $0.88(3)$ \\
$\mathrm{P} 1-\mathrm{C} 1$ & $1.7974(17)$ & $\mathrm{C} 5-\mathrm{C} 6$ & $1.526(2)$
\end{tabular}




\begin{tabular}{|c|c|c|c|}
\hline $\mathrm{N} 1-\mathrm{C} 10$ & $1.350(3)$ & $\mathrm{C} 5-\mathrm{H} 5 \mathrm{~A}$ & 0.9700 \\
\hline $\mathrm{N} 1-\mathrm{C} 14$ & $1.352(3)$ & $\mathrm{C} 5-\mathrm{H} 5 \mathrm{~B}$ & 0.9700 \\
\hline $\mathrm{N} 1-\mathrm{H} 1$ & $0.96(2)$ & $\mathrm{C} 6-\mathrm{C} 7$ & $1.527(2)$ \\
\hline $\mathrm{C} 1-\mathrm{C} 2$ & $1.534(2)$ & C6-H6A & 0.9700 \\
\hline $\mathrm{C} 1-\mathrm{H} 1 \mathrm{~A}$ & 0.9700 & C6-H6B & 0.9700 \\
\hline $\mathrm{C} 1-\mathrm{H} 1 \mathrm{~B}$ & 0.9700 & $\mathrm{C} 7-\mathrm{C} 8$ & $1.530(2)$ \\
\hline $\mathrm{P} 2-\mathrm{O} 6$ & $1.5045(13)$ & C7-H7A & 0.9700 \\
\hline $\mathrm{P} 2-\mathrm{O} 4$ & $1.5535(13)$ & C7-H7B & 0.9700 \\
\hline $\mathrm{P} 2-\mathrm{O} 5$ & $1.5601(13)$ & $\mathrm{C} 8-\mathrm{C} 9$ & $1.537(2)$ \\
\hline $\mathrm{P} 2-\mathrm{C} 9$ & $1.7880(17)$ & $\mathrm{C} 8-\mathrm{H} 8 \mathrm{~A}$ & 0.9700 \\
\hline $\mathrm{N} 2-\mathrm{C} 12$ & $1.324(2)$ & $\mathrm{C} 8-\mathrm{H} 8 \mathrm{~B}$ & 0.9700 \\
\hline $\mathrm{N} 2-\mathrm{H} 21$ & $0.88(3)$ & C9-H9A & 0.9700 \\
\hline $\mathrm{N} 2-\mathrm{H} 22$ & $0.90(3)$ & C9-H9B & 0.9700 \\
\hline $\mathrm{C} 2-\mathrm{C} 3$ & $1.530(2)$ & $\mathrm{C} 10-\mathrm{C} 11$ & $1.365(3)$ \\
\hline $\mathrm{C} 2-\mathrm{H} 2 \mathrm{~A}$ & 0.9700 & $\mathrm{C} 10-\mathrm{H} 10$ & 0.9300 \\
\hline $\mathrm{C} 2-\mathrm{H} 2 \mathrm{~B}$ & 0.9700 & $\mathrm{C} 11-\mathrm{C} 12$ & $1.415(3)$ \\
\hline $\mathrm{O} 3-\mathrm{H} 3$ & $0.78(3)$ & $\mathrm{C} 11-\mathrm{H} 11$ & 0.9300 \\
\hline $\mathrm{C} 3-\mathrm{C} 4$ & $1.523(2)$ & $\mathrm{C} 12-\mathrm{C} 13$ & $1.425(2)$ \\
\hline $\mathrm{C} 3-\mathrm{H} 3 \mathrm{~A}$ & 0.9700 & $\mathrm{C} 13-\mathrm{C} 14$ & $1.359(3)$ \\
\hline $\mathrm{C} 3-\mathrm{H} 3 \mathrm{~B}$ & 0.9700 & $\mathrm{C} 13-\mathrm{H} 13$ & 0.9300 \\
\hline $\mathrm{O} 4-\mathrm{H} 4$ & $0.91(3)$ & C14-H14 & 0.9300 \\
\hline $\mathrm{C} 4-\mathrm{C} 5$ & $1.527(2)$ & & \\
\hline $\mathrm{O} 1-\mathrm{P} 1-\mathrm{O} 2$ & $116.41(8)$ & $\mathrm{C} 6-\mathrm{C} 5-\mathrm{C} 4$ & $114.77(15)$ \\
\hline $\mathrm{O} 1-\mathrm{P} 1-\mathrm{O} 3$ & $105.96(8)$ & $\mathrm{C} 6-\mathrm{C} 5-\mathrm{H} 5 \mathrm{~A}$ & 108.6 \\
\hline $\mathrm{O} 2-\mathrm{P} 1-\mathrm{O} 3$ & $108.76(7)$ & $\mathrm{C} 4-\mathrm{C} 5-\mathrm{H} 5 \mathrm{~A}$ & 108.6 \\
\hline $\mathrm{O} 1-\mathrm{P} 1-\mathrm{C} 1$ & $109.09(8)$ & C6- $55-\mathrm{H} 5 \mathrm{~B}$ & 108.6 \\
\hline $\mathrm{O} 2-\mathrm{P} 1-\mathrm{C} 1$ & $109.62(7)$ & $\mathrm{C} 4-\mathrm{C} 5-\mathrm{H} 5 \mathrm{~B}$ & 108.6 \\
\hline $\mathrm{O} 3-\mathrm{P} 1-\mathrm{C} 1$ & $106.51(8)$ & $\mathrm{H} 5 \mathrm{~A}-\mathrm{C} 5-\mathrm{H} 5 \mathrm{~B}$ & 107.6 \\
\hline $\mathrm{C} 10-\mathrm{N} 1-\mathrm{C} 14$ & $120.49(16)$ & $\mathrm{C} 5-\mathrm{C} 6-\mathrm{C} 7$ & $112.06(14)$ \\
\hline $\mathrm{C} 10-\mathrm{N} 1-\mathrm{H} 1$ & $121.8(15)$ & $\mathrm{C} 5-\mathrm{C} 6-\mathrm{H} 6 \mathrm{~A}$ & 109.2 \\
\hline $\mathrm{C} 14-\mathrm{N} 1-\mathrm{H} 1$ & $117.6(15)$ & $\mathrm{C} 7-\mathrm{C} 6-\mathrm{H} 6 \mathrm{~A}$ & 109.2 \\
\hline $\mathrm{C} 2-\mathrm{C} 1-\mathrm{P} 1$ & $113.66(12)$ & $\mathrm{C} 5-\mathrm{C} 6-\mathrm{H} 6 \mathrm{~B}$ & 109.2 \\
\hline $\mathrm{C} 2-\mathrm{C} 1-\mathrm{H} 1 \mathrm{~A}$ & 108.8 & $\mathrm{C} 7-\mathrm{C} 6-\mathrm{H} 6 \mathrm{~B}$ & 109.2 \\
\hline $\mathrm{P} 1-\mathrm{C} 1-\mathrm{H} 1 \mathrm{~A}$ & 108.8 & $\mathrm{H} 6 \mathrm{~A}-\mathrm{C} 6-\mathrm{H} 6 \mathrm{~B}$ & 107.9 \\
\hline $\mathrm{C} 2-\mathrm{C} 1-\mathrm{H} 1 \mathrm{~B}$ & 108.8 & $\mathrm{C} 6-\mathrm{C} 7-\mathrm{C} 8$ & $114.51(14)$ \\
\hline $\mathrm{P} 1-\mathrm{C} 1-\mathrm{H} 1 \mathrm{~B}$ & 108.8 & $\mathrm{C} 6-\mathrm{C} 7-\mathrm{H} 7 \mathrm{~A}$ & 108.6 \\
\hline $\mathrm{H} 1 \mathrm{~A}-\mathrm{C} 1-\mathrm{H} 1 \mathrm{~B}$ & 107.7 & $\mathrm{C} 8-\mathrm{C} 7-\mathrm{H} 7 \mathrm{~A}$ & 108.6 \\
\hline $\mathrm{O} 6-\mathrm{P} 2-\mathrm{O} 4$ & $113.40(7)$ & $\mathrm{C} 6-\mathrm{C} 7-\mathrm{H} 7 \mathrm{~B}$ & 108.6 \\
\hline $\mathrm{O} 6-\mathrm{P} 2-\mathrm{O} 5$ & $109.15(7)$ & $\mathrm{C} 8-\mathrm{C} 7-\mathrm{H} 7 \mathrm{~B}$ & 108.6 \\
\hline $\mathrm{O} 4-\mathrm{P} 2-\mathrm{O} 5$ & $108.70(7)$ & $\mathrm{H} 7 \mathrm{~A}-\mathrm{C} 7-\mathrm{H} 7 \mathrm{~B}$ & 107.6 \\
\hline $\mathrm{O} 6-\mathrm{P} 2-\mathrm{C} 9$ & $111.12(8)$ & $\mathrm{C} 7-\mathrm{C} 8-\mathrm{C} 9$ & $111.67(14)$ \\
\hline $\mathrm{O} 4-\mathrm{P} 2-\mathrm{C} 9$ & $105.43(8)$ & $\mathrm{C} 7-\mathrm{C} 8-\mathrm{H} 8 \mathrm{~A}$ & 109.3 \\
\hline $\mathrm{O} 5-\mathrm{P} 2-\mathrm{C} 9$ & $108.89(8)$ & $\mathrm{C} 9-\mathrm{C} 8-\mathrm{H} 8 \mathrm{~A}$ & 109.3 \\
\hline $\mathrm{C} 12-\mathrm{N} 2-\mathrm{H} 21$ & $119.9(18)$ & $\mathrm{C} 7-\mathrm{C} 8-\mathrm{H} 8 \mathrm{~B}$ & 109.3 \\
\hline $\mathrm{C} 12-\mathrm{N} 2-\mathrm{H} 22$ & $119.6(16)$ & $\mathrm{C} 9-\mathrm{C} 8-\mathrm{H} 8 \mathrm{~B}$ & 109.3 \\
\hline $\mathrm{H} 21-\mathrm{N} 2-\mathrm{H} 22$ & $120(2)$ & $\mathrm{H} 8 \mathrm{~A}-\mathrm{C} 8-\mathrm{H} 8 \mathrm{~B}$ & 107.9 \\
\hline $\mathrm{C} 3-\mathrm{C} 2-\mathrm{C} 1$ & $112.06(14)$ & $\mathrm{C} 8-\mathrm{C} 9-\mathrm{P} 2$ & $113.70(12)$ \\
\hline
\end{tabular}




\begin{tabular}{|c|c|c|c|}
\hline $\mathrm{C} 3-\mathrm{C} 2-\mathrm{H} 2 \mathrm{~A}$ & 109.2 & $\mathrm{C} 8-\mathrm{C} 9-\mathrm{H} 9 \mathrm{~A}$ & 108.8 \\
\hline $\mathrm{C} 1-\mathrm{C} 2-\mathrm{H} 2 \mathrm{~A}$ & 109.2 & $\mathrm{P} 2-\mathrm{C} 9-\mathrm{H} 9 \mathrm{~A}$ & 108.8 \\
\hline $\mathrm{C} 3-\mathrm{C} 2-\mathrm{H} 2 \mathrm{~B}$ & 109.2 & $\mathrm{C} 8-\mathrm{C} 9-\mathrm{H} 9 \mathrm{~B}$ & 108.8 \\
\hline $\mathrm{C} 1-\mathrm{C} 2-\mathrm{H} 2 \mathrm{~B}$ & 109.2 & $\mathrm{P} 2-\mathrm{C} 9-\mathrm{H} 9 \mathrm{~B}$ & 108.8 \\
\hline $\mathrm{H} 2 \mathrm{~A}-\mathrm{C} 2-\mathrm{H} 2 \mathrm{~B}$ & 107.9 & $\mathrm{H} 9 \mathrm{~A}-\mathrm{C} 9-\mathrm{H} 9 \mathrm{~B}$ & 107.7 \\
\hline $\mathrm{P} 1-\mathrm{O} 3-\mathrm{H} 3$ & $115(2)$ & $\mathrm{N} 1-\mathrm{C} 10-\mathrm{C} 11$ & $120.95(18)$ \\
\hline $\mathrm{C} 4-\mathrm{C} 3-\mathrm{C} 2$ & $113.89(15)$ & $\mathrm{N} 1-\mathrm{C} 10-\mathrm{H} 10$ & 119.5 \\
\hline $\mathrm{C} 4-\mathrm{C} 3-\mathrm{H} 3 \mathrm{~A}$ & 108.8 & $\mathrm{C} 11-\mathrm{C} 10-\mathrm{H} 10$ & 119.5 \\
\hline $\mathrm{C} 2-\mathrm{C} 3-\mathrm{H} 3 \mathrm{~A}$ & 108.8 & $\mathrm{C} 10-\mathrm{C} 11-\mathrm{C} 12$ & $120.38(18)$ \\
\hline $\mathrm{C} 4-\mathrm{C} 3-\mathrm{H} 3 \mathrm{~B}$ & 108.8 & $\mathrm{C} 10-\mathrm{C} 11-\mathrm{H} 11$ & 119.8 \\
\hline $\mathrm{C} 2-\mathrm{C} 3-\mathrm{H} 3 \mathrm{~B}$ & 108.8 & $\mathrm{C} 12-\mathrm{C} 11-\mathrm{H} 11$ & 119.8 \\
\hline $\mathrm{H} 3 \mathrm{~A}-\mathrm{C} 3-\mathrm{H} 3 \mathrm{~B}$ & 107.7 & $\mathrm{~N} 2-\mathrm{C} 12-\mathrm{C} 11$ & $121.92(17)$ \\
\hline $\mathrm{P} 2-\mathrm{O} 4-\mathrm{H} 4$ & $113(2)$ & $\mathrm{N} 2-\mathrm{C} 12-\mathrm{C} 13$ & $121.32(17)$ \\
\hline $\mathrm{C} 3-\mathrm{C} 4-\mathrm{C} 5$ & $112.68(15)$ & $\mathrm{C} 11-\mathrm{C} 12-\mathrm{C} 13$ & $116.75(16)$ \\
\hline $\mathrm{C} 3-\mathrm{C} 4-\mathrm{H} 4 \mathrm{~A}$ & 109.1 & $\mathrm{C} 14-\mathrm{C} 13-\mathrm{C} 12$ & $119.77(17)$ \\
\hline $\mathrm{C} 5-\mathrm{C} 4-\mathrm{H} 4 \mathrm{~A}$ & 109.1 & $\mathrm{C} 14-\mathrm{C} 13-\mathrm{H} 13$ & 120.1 \\
\hline $\mathrm{C} 3-\mathrm{C} 4-\mathrm{H} 4 \mathrm{~B}$ & 109.1 & $\mathrm{C} 12-\mathrm{C} 13-\mathrm{H} 13$ & 120.1 \\
\hline $\mathrm{C} 5-\mathrm{C} 4-\mathrm{H} 4 \mathrm{~B}$ & 109.1 & $\mathrm{~N} 1-\mathrm{C} 14-\mathrm{C} 13$ & $121.60(18)$ \\
\hline $\mathrm{H} 4 \mathrm{~A}-\mathrm{C} 4-\mathrm{H} 4 \mathrm{~B}$ & 107.8 & $\mathrm{~N} 1-\mathrm{C} 14-\mathrm{H} 14$ & 119.2 \\
\hline $\mathrm{P} 2-\mathrm{O} 5-\mathrm{H} 5$ & $117(2)$ & $\mathrm{C} 13-\mathrm{C} 14-\mathrm{H} 14$ & 119.2 \\
\hline $\mathrm{O} 1-\mathrm{P} 1-\mathrm{C} 1-\mathrm{C} 2$ & $74.30(14)$ & $\mathrm{O} 6-\mathrm{P} 2-\mathrm{C} 9-\mathrm{C} 8$ & $66.99(14)$ \\
\hline $\mathrm{O} 2-\mathrm{P} 1-\mathrm{C} 1-\mathrm{C} 2$ & $-54.24(14)$ & $\mathrm{O} 4-\mathrm{P} 2-\mathrm{C} 9-\mathrm{C} 8$ & $-56.25(14)$ \\
\hline $\mathrm{O} 3-\mathrm{P} 1-\mathrm{C} 1-\mathrm{C} 2$ & $-171.74(12)$ & $\mathrm{O} 5-\mathrm{P} 2-\mathrm{C} 9-\mathrm{C} 8$ & $-172.75(12)$ \\
\hline $\mathrm{P} 1-\mathrm{C} 1-\mathrm{C} 2-\mathrm{C} 3$ & $-167.86(12)$ & $\mathrm{C} 14-\mathrm{N} 1-\mathrm{C} 10-\mathrm{C} 11$ & $1.9(3)$ \\
\hline $\mathrm{C} 1-\mathrm{C} 2-\mathrm{C} 3-\mathrm{C} 4$ & $-176.88(14)$ & $\mathrm{N} 1-\mathrm{C} 10-\mathrm{C} 11-\mathrm{C} 12$ & $-0.4(3)$ \\
\hline $\mathrm{C} 2-\mathrm{C} 3-\mathrm{C} 4-\mathrm{C} 5$ & $-178.52(15)$ & $\mathrm{C} 10-\mathrm{C} 11-\mathrm{C} 12-\mathrm{N} 2$ & $176.98(19)$ \\
\hline $\mathrm{C} 3-\mathrm{C} 4-\mathrm{C} 5-\mathrm{C} 6$ & $-179.83(15)$ & $\mathrm{C} 10-\mathrm{C} 11-\mathrm{C} 12-\mathrm{C} 13$ & $-1.7(3)$ \\
\hline $\mathrm{C} 4-\mathrm{C} 5-\mathrm{C} 6-\mathrm{C} 7$ & $-179.62(14)$ & $\mathrm{N} 2-\mathrm{C} 12-\mathrm{C} 13-\mathrm{C} 14$ & $-176.38(18)$ \\
\hline $\mathrm{C} 5-\mathrm{C} 6-\mathrm{C} 7-\mathrm{C} 8$ & $-177.72(15)$ & $\mathrm{C} 11-\mathrm{C} 12-\mathrm{C} 13-\mathrm{C} 14$ & $2.3(3)$ \\
\hline $\mathrm{C} 6-\mathrm{C} 7-\mathrm{C} 8-\mathrm{C} 9$ & $-177.78(14)$ & $\mathrm{C} 10-\mathrm{N} 1-\mathrm{C} 14-\mathrm{C} 13$ & $-1.3(3)$ \\
\hline $\mathrm{C} 7-\mathrm{C} 8-\mathrm{C} 9-\mathrm{P} 2$ & $-169.70(12)$ & $\mathrm{C} 12-\mathrm{C} 13-\mathrm{C} 14-\mathrm{N} 1$ & $-0.9(3)$ \\
\hline
\end{tabular}

Hydrogen-bond geometry $\left(\AA,{ }^{\circ}\right)$

\begin{tabular}{lllll}
\hline$D-\mathrm{H} \cdots A$ & $D-\mathrm{H}$ & $\mathrm{H} \cdots A$ & $D \cdots A$ & $D-\mathrm{H} \cdots A$ \\
\hline $\mathrm{O} 3-\mathrm{H} 3 \cdots \mathrm{O} 66^{\mathrm{i}}$ & $0.78(3)$ & $1.85(3)$ & $2.6171(18)$ & $166(3)$ \\
$\mathrm{O} 5-\mathrm{H} 5 \cdots 1^{\mathrm{ii}}$ & $0.88(3)$ & $1.64(3)$ & $2.5059(18)$ & $168(3)$ \\
$\mathrm{O} 4-\mathrm{H} 4 \cdots \mathrm{O} 2^{\mathrm{iii}}$ & $0.91(3)$ & $1.59(3)$ & $2.4977(17)$ & $178(3)$ \\
$\mathrm{N} 1-\mathrm{H} 1 \cdots \mathrm{O} 6$ & $0.96(2)$ & $1.74(3)$ & $2.696(2)$ & $173(2)$ \\
$\mathrm{N} 2-\mathrm{H} 22 \cdots \mathrm{O} 2^{\mathrm{iv}}$ & $0.90(3)$ & $1.92(3)$ & $2.806(2)$ & $170(2)$ \\
$\mathrm{N} 2-\mathrm{H} 21 \cdots{ }^{v}$ & $0.88(3)$ & $2.14(3)$ & $2.965(2)$ & $156(3)$ \\
\hline
\end{tabular}

Symmetry codes: (i) $-x+1,-y+2,-z+1$; (ii) $-x,-y+1,-z+1$; (iii) $-x+1,-y+1,-z+1$; (iv) $x, y-1, z+1$; (v) $x+1, y-1, z+1$. 